\title{
Acceso y uso de los mercados financieros para el pago de los gastos de la educación básica
}

\author{
Access and use of financial markets for basic education expenses \\ Kristiano Raccanello ${ }^{\text {a,* }}$, Laura Elena Carrillo Cubillas ${ }^{b}$ \\ y Mariana Guzmán Yerena ${ }^{\mathrm{C}}$ \\ ${ }^{a}$ El Colegio de Tlaxcala A.C.

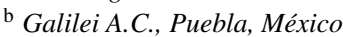 \\ ${ }^{\mathrm{c}}$ Deloitte, Ciudad de México
}

Recibido el 5 de mayo de 2015; aceptado el 17 de marzo de 2016

Disponible en Internet el 12 de mayo de 2017

\section{Resumen}

A raíz de la crisis financiera del 2008, el ajuste macroeconómico ha afectado a los hogares mexicanos a través de un mayor desempleo y una caída del poder adquisitivo. También, las familias tenían que seguir financiando los gastos en educación, a veces endeudándose en el mercado financiero formal o informal. La hipótesis de este artículo propone que el acceso y el uso de los productos financieros formales e informales permiten reducir los problemas financieros asociados con los gastos escolares en los niveles de educación básica. El modelo probit se estima con una muestra constituida por cuatrocientos hogares que residen en el municipio de San Pedro Cholula (estado de Puebla). Los resultados indican que un empeoramiento de las condiciones laborales y la pérdida del empleo se asocian con una mayor probabilidad de enfrentar dificultades financieras. Asimismo, el acceso al mercado financiero formal permite reducir la probabilidad de enfrentar estas dificultades, mientras que para el informal los resultados están en función de las características de cada intermediario financiero.

(C) 2017 Universidad Nacional Autónoma de México, Facultad de Contaduría y Administración. Este es un artículo Open Access bajo la licencia CC BY-NC-ND (http://creativecommons.org/licenses/by-nc-nd/4.0/).

Palabras clave: Financiamiento de la educación; Gasto privado; Mercados financieros; México

Códigos JEL: $\mathrm{I} 22$

\footnotetext{
* Autor para correspondencia.

Correo electrónico: kristiano.raccanello@ coltlax.edu.mx (K. Raccanello).

La revisión por pares es responsabilidad de la Universidad Nacional Autónoma de México.
} 


\begin{abstract}
The aftermath of the 2008 financial crisis and the macroeconomic adjustment that followed provided higher unemployment and eroded the Mexican households' purchasing power. Though, families had to continue financing education expenditures, sometimes borrowing in the formal or informal financial market. The hypothesis of this paper posits that access and use of formal and informal financial products can reduce the financial problems associated with basic school private expenditures. The probit model is estimated through a sample of four hundred households drawn from the municipality of San Pedro Cholula (state of Puebla). The results indicate that a worsening of working conditions and sudden unemployment are associated with an increased likelihood of difficulties in making ends meet. Moreover, accessing the formal financial market lessens the likelihood of such problems, but for the informal counterpart results depend on the characteristics of each financial intermediary.

(C) 2017 Universidad Nacional Autónoma de México, Facultad de Contaduría y Administración. This is an open access article under the CC BY-NC-ND license (http://creativecommons.org/licenses/by-nc-nd/4.0/).
\end{abstract}

Keywords: Education financing; Financial markets; Private spending; Mexico

JEL classification: $\mathrm{I} 22$

\title{
Antecedentes
}

La educación puede ser considerada de distintas formas: a) como un bien de consumo que produce utilidad para los individuos sin tener ningún tipo de incidencia en la productividad; b) como un medio que permite identificar y calificar destrezas y habilidades, o bien c) como un medio para incrementar el capital humano (Carrillo, 2006), el cual, a su vez, se considera un factor productivo dada la relación que existe entre el nivel de conocimientos de los trabajadores con su capacidad para utilizarlos y, por ende, su productividad (Serrano, 1996). De acuerdo a esta última perspectiva, el gasto en educación es una inversión que genera mejores condiciones de vida para el individuo que la recibe. En este sentido, a nivel individual, la educación es un proceso que contribuye al desarrollo de cada ser humano mediante el cual es posible acceder a mayores niveles de ingresos y mejores servicios, mientras que a nivel colectivo hay un consenso general en considerarla como una palanca de desarrollo social (Marcel y Tokman, 2005).

En relación con lo anterior, existe una estrecha relación entre la escolaridad y el nivel de ingresos de los individuos (Becker, 1964; Mincer, 1974), con evidentes beneficios intergeneracionales que se asocian con los niveles de instrucción y riqueza de las generaciones futuras (Hoyos, Martínez y Székely, 2010).

Por otro lado, se tiene que en México el porcentaje del ingreso destinado a la educación disminuye conforme aumenta el ingreso de los hogares, con la excepción del último decil (Instituto Nacional de Estadística y Geografía [INEGI, 2010]); es decir, «los gastos en educación por parte de los estratos más pobres son mucho menores que los correspondientes a los estratos altos. Sin embargo, [...] los estratos bajos de la sociedad [...] concentran sus gastos en el nivel básico, mientras que las clases altas lo hacen en educación superior» (Bracho, 1995, pp. 2-3, citando a Llamas, 1993). Asimismo, también se observa que las personas en los hogares que pertenecen a los estratos con menores ingresos estudian menos años y en peores condiciones respecto a las de ingresos más altos (Hochschild y Scovronick, 2004). 


\section{Educación, capital humano y resultados económicos}

La importancia de la educación a nivel individual radica en ser un proceso permanente que contribuye al desarrollo del ser humano, así como el medio principal para obtener, transmitir y acrecentar la cultura, siendo un factor determinante en la adquisición de conocimiento y de la formación personal. El capital humano, que de acuerdo a Becker (1964) se define como el «[...] conjunto de las capacidades productivas que un individuo adquiere por acumulación de conocimientos generales o específicos», es el puente entre la educación y el trabajo, siendo este último la concretización de lo aprendido en la etapa escolar, fortalecido por la experiencia y la práctica cotidiana del quehacer laboral.

Los primeros años de vida y el adecuado desarrollo de las personas resultan cruciales para su formación como individuos productivos. En estas etapas los cuidados de los padres, el acceso a una alimentación balanceada y a servicios médicos, así como la posibilidad de obtener una educación oportuna y de calidad, permiten que, a nivel intergeneracional, los individuos puedan alcanzar mejores niveles de vida a través de un círculo virtuoso (Raccanello, 2012).

Una de las manifestaciones a través de las cuales puede apreciarse este aspecto consiste en los resultados económicos de las microempresas, que en México representan el $95.6 \%$ de las unidades productivas y que emplean al 45.6\% de la fuerza laboral (Pavón, 2010) pero que aportan solo el 15\% del producto interno bruto (PIB) (Secretaría de Economía, 2010). En relación con esto, se tiene que conforme el grado de estudios del microempresario aumenta, se observa un incremento paulatino en las utilidades operativas respecto a los que tienen solo educación primaria ${ }^{1}$, concluyéndose que «a mayor educación se espera que el microempresario tenga competencias que, conjuntamente a una mayor comprensión de las problemáticas, le permitan realizar análisis y tomar mejores decisiones que se reflejan en resultados positivos para la microempresa» (Raccanello y Saucedo, 2015, p. 17). Estos resultados se sustentan también con los datos que publican PROMÉXICO $(2014)^{2}$ y el Banco de Información Económica (INEGI, 2014), en donde la correlación entre los años promedio de escolaridad por entidad federativa con el índice de productividad es del $68.9 \%$, y con respecto al promedio de la utilidad bruta por actividad económica es del $41.5 \%$ para manufactura y del $39 \%$ para comercio y servicios.

\section{Educación en México}

Frente a los resultados que han demostrado las carencias del sistema educativo mexicano a nivel internacional (OCDE/PISA, 2000, 2003, 2006, 2009), este ha sido objeto de una importante reforma aprobada en diciembre de 2012 y promulgada en febrero de 2013.

Aun cuando sea demasiado pronto para poder determinar el impacto de la reforma educativa, las medidas que habían sido tomadas por el Estado antes de la reforma ya reflejaban un aumento de la tasa bruta de matriculación (o cobertura) ${ }^{3}$ en educación primaria y secundaria (Secretaría de Educación Pública [SEP, 2010]), en donde para la educación básica las cifras reportadas por la SEP indican que a nivel nacional, durante el ciclo escolar 1990-1991, existía un 95.9\% de cobertura para el nivel de primaria, que aumentó al 98.6\% para el ciclo escolar 2009-2010. Asimismo, a

\footnotetext{
${ }^{1}$ Secundaria: $+1,188$ pesos; preparatoria: $+1,447$ pesos; universidad: $+2,332$ pesos.

2 Principales indicadores de inversión y comercio para los Estados de la República (2014).

${ }^{3}$ La tasa bruta de matriculación (o cobertura) se calcula como: [(matrícula total de un nivel educativo, sin importar la edad) / (población total que tiene la edad que corresponde al nivel educativo)] $* 100$.
} 
nivel de secundaria, considerando que en el ciclo 1990-1991 este no era obligatorio, la cobertura nacional aumentó del 68 al 95.3\% (ciclo escolar 2009-2010).

De acuerdo a cifras publicadas por la UNESCO (2010a), en 2009 la tasa neta ${ }^{4}$ de matriculación escolar a nivel de primaria era del $93.9 \%$ (niños entre 6 y 12 años de edad), del $67.2 \%$ para la secundaria (adolescentes de 12 a 15 años de edad) pero tan solo el $25.7 \%$ de los jóvenes se inscribía en preparatoria o bachillerato. Estos porcentajes reflejan que a mayor nivel de escolaridad, mayor es la diferencia entre la tasa bruta y la tasa neta de matriculación, es decir, mayor es el porcentaje de la población inscrita que no cumple con la edad correspondiente a ese grado académico. Adicionalmente, debe señalarse que la educación secundaria y la educación media aún no son alcanzables para un sector importante de la población, y muchos de los jóvenes que ingresan a estos niveles escolares desertan ${ }^{5}$ y no concluyen sus estudios (SEP, 2011).

\section{Causas de la deserción escolar}

La literatura que ha analizado las causas asociadas con la deserción escolar señala que esta se debe principalmente a la falta de recursos económicos para cubrir los costos directos (inscripción y colegiaturas) e indirectos (materiales de trabajo, uniformes y transporte) para poder asistir, así como a la percepción de una baja calidad educativa (Rumberger, 1995; Morrisson, 2002; Ersado, 2005; Yuren, de la Cruz, Cruz, Araújo-Olivera y Estrada, 2005; Baschieri y Falkingham, 2007; Rosati y Rossi, 2007). Los estudiantes que tienen menores habilidades son los más propensos a abandonar la escuela (Bacolod y Ranjan, 2008), y realizar actividades laborales también puede perjudicar la asistencia, los resultados escolares y, en última instancia, la permanencia en la institución educativa (Basu y Van, 1998; Basu, 1999; Orazem y Gunnarsson, 2003; Beegle, Dehejia y Gatti, 2005; Edmonds y Pavcnik, 2005; Gunnarsson, Orazem y Sánchez, 2006; Bhalotra, 2007).

La crisis financiera que sacudió a la economía mundial en el año 2008 ha modificado la distribución de los presupuestos nacionales. Según la UNESCO (2011), muchos de los países más pobres del mundo se han visto afectados en sus planes para el financiamiento de la educación replanteando sus metas para el 2015. En México, el porcentaje del PIB destinado a la educación aumentó del 4.7\% durante los años 2006-2007 al 4.9\% en 2008, para alcanzar el 5.2\% en el trienio 2009-2011 (UNESCO, 2010a). A pesar de este aumento, la falta de empleo y la pérdida de poder adquisitivo consecuentes a la crisis financiera podrían generar presiones y problemáticas económicas en los hogares. Si bien el gasto en educación es el resultado de un proceso de asignación de recursos que refleja una estructura de prioridades y objetivos sociales, familiares e individuales (Bracho, 1995), este debe también tomar en cuenta los recursos económicos de los cuales dispone el hogar.

Para ello, la crisis económica de 2008 repercutió en un menor crecimiento del PIB (1.2\%), comparado con el año anterior (3.3\% en el año 2007), pero con una caída en 2009 (-6.1\%), seguida por una parcial recuperación (5.5\%) en 2010 (Banco de México, 2011). La caída de la producción fue acompañada por un aumento de la inflación, que redujo el poder adquisitivo de los salarios, y por un aumento de la tasa de desocupación abierta principalmente en 2009, misma que en 2010 todavía no lograba reducirse a los niveles previos a la crisis. Este incremento en la

\footnotetext{
${ }^{4}$ La tasa neta de matriculación escolar se calcula como: [(matrícula correspondiente al rango de edad de un nivel educativo) / (población total de la edad correspondiente al nivel educativo)] * 100 .

5 Deserción en primaria (2009-2010): 0.9\%. Deserción en secundaria (2009-2010): 6.2\%. Deserción en educación media (2009-2010): 15.6\% (SEP, 2011).
} 
tasa de desocupación abierta afectó a ambos géneros; para los hombres aumentó del 3.9\% (2008) al 5.5\% (2009) y se estableció en 5.4\% en el año 2010. De igual forma, para las mujeres pasó del $4.1 \%$ en 2008 al $5.5 \%$ en 2009, estableciéndose en el 5.4\% para el 2010 (Botello, 2011). Además de un aumento en el desempleo, la población ocupada percibió menores salarios (Botello, 2011, cuadro 6). Derivado de lo anterior, podría anticiparse que estos ajustes a nivel macroeconómico afectan a las familias, en particular si una de las personas que proveían recursos al hogar perdió el empleo o si logró reubicarse, dependiendo de las nuevas condiciones laborales.

En este artículo se asocian los efectos de la crisis económica con el haber enfrentado alguna dificultad para cubrir los gastos en educación, situación ya puntualizada por la UNESCO (2010b) en su «Informe de Seguimiento de la Educación para Todos en el Mundo» al observar una reducción de los recursos destinados para cubrir este rubro, a pesar de que el gasto por estudiante en primaria y secundaria muestra una tendencia alcista en los años posteriores a la crisis financiera (UNESCO, 2010a). Una explicación a esta tendencia es que los hogares pudieran haber acudido a los mercados de crédito formal o informal para poder financiar estos gastos; la Encuesta Nacional de Inclusión Financiera (SHCP, CNBV e INEGI, 2012) presenta evidencia en este sentido. A pesar de que los gastos en educación se encuentren agrupados en la categoría «gastos de educación o salud», lo que hace imposible aislar la variable de interés, del 38.6\% de la población que había ahorrado en el último año (2011), el 29.55\% destinó esos recursos a gastos en educación o en salud. Del mismo modo, el $35.96 \%$ solicitó créditos informales, de los cuales el 32.93\% también destinó ese dinero a gastos en educación (o salud). En cuanto al financiamiento formal se refiere, el $30.76 \%$ utilizó el dinero prestado para este mismo fin.

Complementando lo anterior, Bray (2007) indica que el gasto en educación puede dividirse en: a) costos de oportunidad, aquellas actividades que los individuos o familias dejan de realizar para invertir en educación, y b) gastos monetarios que pueden realizarse «dentro» (cooperaciones y cuotas escolares) y «fuera» de la escuela (compra de uniformes, papelería, transporte y trámites). En los sistemas educativos de los países industrializados los gastos «dentro» de la escuela son cubiertos en su totalidad por los gobiernos y los gastos «fuera» de la escuela constituyen una pequeña proporción del gasto total de los hogares (Santibáñez, Campos y Jarillo, 2011). Sin embargo, en los países donde la capacidad del Estado para financiar la educación pública es limitada, las familias deben realizar esfuerzos para co-financiar la educación de sus integrantes (OECD y UNESCO, 2002).

En relación con la entidad federativa objeto de este artículo — Puebla—, está catalogada como el sexto estado con menor escolaridad; sus habitantes en promedio cuentan con 7.9 años de escolaridad (inferior a la media nacional: 8.7 años) y presentan altos niveles de deserción para los niveles de primaria (1.2\%) y secundaria (4.7\%) (SEP, 2012). Específicamente, para el municipio de San Pedro Cholula, donde se realizó el estudio empírico, se observa una mayor deserción que a nivel estatal, tanto a nivel de primaria como de secundaria, con 1.3 y $5.2 \%$, respectivamente (SEP, 2012).

\section{Créditos formales e informales}

El acceso y el uso de los servicios financieros pueden proporcionar una mejora en el bienestar de los hogares. En particular, a través del acceso los hogares pueden «[...] movilizar ahorros, recibir créditos, administrar riesgos y participar en los sistemas de pagos» (CNBV, 2009), mientras que la componente uso estará determinada por aquel porcentaje de la población que utiliza alguno de estos productos o servicios. Sin embargo, no toda la población utiliza estos servicios, y es 
esta la que se considera como financieramente excluida, representando un importante mercado potencial para los intermediarios financieros informales.

En este sentido, la Comisión Nacional para la Protección y Defensa de los Usuarios de Servicios Financieros (CONDUSEF) indica que: «[...] para la mayor parte de la población mexicana existen dos sistemas financieros: el formal y el informal. El primero suele estar casi siempre fuera de su alcance; el segundo, en el que se realiza la mayor parte de su actividad financiera, se desarrolla en un ámbito comúnmente sin supervisión e inadvertida en registros y estadísticas oficiales» (CONDUSEF, 2013), argumentando que la actividad financiera informal es una fuente de crédito importante conformada principalmente por prestamistas y casas de empeño.

En el año 2009, según el Primer Reporte de Inclusión Financiera (CNBV, 2009), Puebla era el tercer estado con menos municipios que contaban con sucursales bancarias y el segundo con menos municipios con cajeros automáticos. De igual forma, era el séptimo estado con menos usuarios de tarjeta de débito (43\%).

En el municipio de San Pedro Cholula, con respecto a los indicadores de uso del crédito formal, había 0.81 sucursales bancarias y la misma proporción de cajeros automáticos, así como 50 puntos de venta por cada 10,000 adultos (CNBV, 2010). Del mismo modo, el $28.53 \%$ de la población económicamente activa contaba con tarjeta de débito y el $4.35 \%$, con tarjeta de crédito. El comparativo con el estado de Puebla y con la media nacional ${ }^{6}$ indica que este municipio tenía una cobertura inferior por cuanto concierne el acceso y el uso del crédito formal (CNBV, 2010, p. 302).

Bajo estas premisas, el objetivo principal de este estudio es analizar si las dificultades que han presentado los hogares para pagar los gastos en educación de los hijos se aminoran con el acceso y el uso de los mercados de crédito formal e informal, al considerar los cambios en la situación laboral que se presentaron en los jefes de familia, en un entorno que estaba sujeto a las secuelas de la crisis financiera del 2008. Para ello, la hipótesis de este trabajo plantea que el uso y el acceso a fuentes de financiamientos, formales e informales, aminora los problemas para solventar los gastos en educación.

\section{Datos y metodología}

\section{Datos}

De un universo de 62 escuelas primarias (40 públicas y 22 privadas) en el municipio de San Pedro Cholula, se seleccionaron las instituciones mediante un muestreo sistemático en dos etapas por conglomerados con un coeficiente de elevación igual a 4 para el muestreo de las instituciones privadas y de 8 para las públicas, con el objetivo de elegir aleatoriamente 5 escuelas de cada conglomerado. Una vez determinadas las escuelas, se seleccionaron por medio de un muestreo probabilístico simple a los 400 estudiantes de primaria para proceder a encuestar a uno de los adultos (preferiblemente el padre o la madre) de los hogares correspondientes. El levantamiento de la información se llevó a cabo entre los meses de enero y junio del año 2011; los datos se terminaron de capturar en octubre, y la revisión de la base de datos concluyó en diciembre del mismo año.

\footnotetext{
61.77 sucursales por cada 10,000 adultos.
} 


\section{Cuestionario}

El instrumento estaba integrado por 51 reactivos cerrados de opción múltiple y dicotómica organizado en tres secciones: a) aspectos socioeconómicos del hogar (estado civil y escolaridad de los padres, personas que aportan ingresos de forma regular e irregular, activos, ingresos, ahorros y deudas del hogar); 2) información concerniente a los hijos (nivel escolar, escuela a la que asisten, becas) y, 3) mecanismos de financiamiento (acceso, uso y características de las fuentes de financiamiento formales e informales).

\section{Estadística descriptiva}

De acuerdo a los resultados de la encuesta, la mayoría de los hogares de la muestra se conforma por parejas $(84.75 \%$, aunque solo el $69 \%$ declaró estar casada), con un promedio de cuatro habitantes por hogar $(40 \%)$ y con dos hijos $(42.75 \%)$. La mayoría de los padres tiene como máximo nivel de estudios de preparatoria (el $46.48 \%$ de los padres y el $39.80 \%$ de las madres) o secundaria (24.62 y $31.74 \%$, respectivamente).

En cuanto a los aspectos económicos, el $49.25 \%$ de los hogares cuenta con solo un miembro que aporta ingresos de forma regular, y en el $17 \%$ de los casos se detectaron aportaciones irregulares. Aunado a lo anterior, el nivel de ingresos de los hogares es bajo: el 59\% de la muestra percibe hasta 4 salarios mínimos (menos de 6,317 pesos mensuales) y el $23.25 \%$ percibe entre 5 y 7 salarios mínimos (entre 6,317 y 11,053 pesos).

Por otro lado, más de la mitad de los hogares (51.25\%) no tiene ahorros y el $33.25 \%$ ha acumulado menos de 11,369 pesos, cantidad que representa un fondo bastante limitado en caso de que ocurra alguna contingencia. En relación con las deudas, el $73.75 \%$ de los encuestados declara deber entre 3,001 y 25,000 pesos, y para los montos superiores los porcentajes se reducen paulatinamente. Adicionalmente, solo el $21 \%$ de los encuestados que declararon tener alguna tarjeta de crédito o departamental manifiestan haber tenido problemas para pagar sus saldos en el año 2008, porcentaje que aumenta al $36.75 \%$ y al $52.75 \%$ en los años siguientes (2009 y 2010, respectivamente), cuya tendencia acompaña la agudización de la crisis financiera y del desempleo durante estos años.

En relación con las actividades laborales, la mayoría de los padres son asalariados por parte del sector privado $(40.75 \%)$, autoempleados $(28 \%) \mathrm{y}$, en menor medida, se desempeñan en el sector público (19.75\%). Durante el último año, el 74\% conservó el mismo empleo, a veces teniendo que aceptar cambios en las condiciones laborales $(7.25 \%)$, o incorporando otra actividad para complementar el nivel de ingresos (6.5\%). De los restantes, el $10.5 \%$ perdió su trabajo lográndose reubicar (6\%) o siguió sin empleo (4.5\%) para la fecha de aplicación de la encuesta.

La situación de las madres es un poco distinta debido a que la mayoría se dedica al hogar (30.5\%); las restantes son auto-empleadas (23.25\%), se desempeñan en la iniciativa privada $(21.25 \%)$ o en el sector público (16\%). Un porcentaje menor de mujeres respecto a los hombres ha conservado su mismo empleo (56.25\%), teniendo que aceptar cambios en las condiciones laborales $(4.5 \%)$. También se detecta un mayor porcentaje de mujeres, respecto a los hombres, que incorpora otras actividades laborales para complementar los ingresos (13\%). El 8.25\% perdió su trabajo, y solo el $2.5 \%$ ha logrado reubicarse, mientras que el restante $5.75 \%$ siguió sin empleo.

En los hogares encuestados se encontró que hay uno (33\%), dos $(40.25 \%)$ o tres $(21.75 \%)$ hijos que actualmente estudian. Los datos revelan que el porcentaje de los que asisten a las escuelas privadas disminuye en la medida en la cual aumenta el número de hijos en el hogar, 
posiblemente por la carga económica que representa mantenerlos en estas instituciones. En relación con esto, el $36.25 \%$ de los hogares afirma haber tenido problemas para pagar los gastos en educación durante el ciclo escolar 2010-2011, tratando de solventarlos pidiendo prestado a familiares $(48.97 \%)$, participando en alguna tanda (15.86\%) o financiándolos con tarjetas de crédito (13.10\%). Frente a estos problemas, los menores dejaron de estudiar solo en el $1.38 \%$ de los casos, mientras que el $2.78 \%$ tuvo que incorporar algún trabajo remunerado a las actividades académicas.

\section{Metodología}

Para poder probar la hipótesis de este trabajo se estimó un modelo probit con errores estándares robustos, en el cual la variable dependiente problema2010_2011 toma valor de 1 si el hogar tuvo problemas económicos para pagar la educación de sus hijos durante el año escolar 2010-2011, y de 0 si no los tuvo. Los coeficientes $d F / d x$ representan los cambios marginales, si son significativos y positivos (negativos), de tener (no tener) problemas para pagar la educación de los hijos. La descripción de cada una de las variables independientes incluidas en el modelo se presenta en el anexo (tabla A.1).

\section{Resultados}

De acuerdo a la prueba Hosmer-Lemeshow, el modelo se ajusta bien a los datos ( $\mathrm{p}=0.7616), \mathrm{y}$ esta especificación permite clasificar correctamente al $84.5 \%$ de las observaciones de la variable dependiente (tabla 1).

Según las estimaciones, los problemas para el pago de la educación presentan un comportamiento inercial, ya que si el hogar tuvo dificultad para enfrentar los gastos educativos en el ciclo 2009-2010, la probabilidad de que el problema ocurra también en el ciclo escolar siguiente es del 42\%, mientras que si el problema se presentó dos ciclos antes (2008-2009) la probabilidad, aun siendo menor, sigue siendo bastante elevada (20.8\%). Evidentemente, esta situación refleja cierto grado de vulnerabilidad por parte de los hogares que no logran eliminar completamente no obstante el paso del tiempo.

Aunque los niveles de educación de ambos padres se incluyeron en el modelo, solo los de la madre se asocian con los problemas para el pago de la educación. Respecto a las que tienen niveles de educación universitaria, estos problemas se verifican con una mayor probabilidad (11.9\%) en los casos en que las madres tienen educación media, probablemente por un manejo deficiente de los recursos. No obstante, si ellas presentan un nivel de escolaridad de primaria o menor, la probabilidad de presentar este problema disminuye en un $26.9 \%$. Este comportamiento se puede explicar a través de las preferencias de los padres al momento de escoger el tipo de institución a la que asistirán sus hijos. Si las madres tienen bajos niveles educativos, podrían enviar sus hijos a escuelas públicas, donde los gastos en educación son menores y el compromiso financiero es también más fácil de cumplir. En todos los modelos estimados a lo largo de esta investigación, el coeficiente asociado a esta variable siempre presentó signo negativo (significativo al $1 \%$ ). Se realizó un análisis de la información muestral, encontrando que de los 30 hogares en donde la madre tiene este nivel escolar, en 27 casos los hijos estaban cursando alguna escuela pública, lo cual apoya la interpretación anterior.

Por otro lado, ninguna de las variables de ingresos resultó estadísticamente significativa, por lo cual no se detectó ninguna asociación entre estas y los problemas para pagar la educación de los 
Tabla 1

Estimaciones modelo probit

\begin{tabular}{|c|c|c|c|c|c|}
\hline \multirow{2}{*}{$\frac{\text { Problema2010_2011 }}{\text { Problema2009_2010 }}$} & \multirow{2}{*}{$\frac{\mathrm{dF} / \mathrm{dx}}{0.420}$} & \multicolumn{2}{|l|}{ EE robusto } & \multirow{2}{*}{$\frac{d F / d x}{-0.080}$} & \multirow{2}{*}{$\begin{array}{l}\text { EE robusto } \\
0.137\end{array}$} \\
\hline & & $0.067^{* * *}$ & Hprep_priv & & \\
\hline Problema2008_2009 & 0.208 & $0.091^{* * *}$ & Hprep_pub & -0.015 & 0.088 \\
\hline Problema2007_2008 & 0.023 & 0.105 & H_esc_privadas & -0.160 & 0.162 \\
\hline P_hastaprimaria & 0.068 & 0.115 & H_esc_públicas & 0.174 & 0.222 \\
\hline Pmedia & -0.051 & 0.074 & Beca_myp_priv & $-2.11 \mathrm{E}-05$ & 8.59E-05 \\
\hline M_hastaprimaria & -0.269 & $0.039^{* * * *}$ & Beca_myp_pub & $-1.11 \mathrm{E}-05$ & $1.28 \mathrm{E}-04$ \\
\hline Mmedia & 0.119 & $0.066^{*}$ & Beca_prim_priv & $-3.75 \mathrm{E}-05$ & $3.75 \mathrm{E}-05$ \\
\hline Aport_regulares & 0.045 & 0.041 & Beca_prim_pub & $-1.81 \mathrm{E}-06$ & 4.28E-05 \\
\hline Aport_irregulares & 0.221 & $0.077^{\text {***** }}$ & Beca_sec_priv & $2.47 \mathrm{E}-05$ & $4.49 \mathrm{E}-05$ \\
\hline Propia & -0.083 & 0.113 & Beca_sec_pub & 8.81E-04 & $6.38 \mathrm{E}-04$ \\
\hline Renta & -0.033 & 0.107 & Beca_prep_priv & $1.17 \mathrm{E}-04$ & $6.20 \mathrm{E}-05^{*}$ \\
\hline Ing_hasta $2 \mathrm{sm}$ & -0.155 & 0.098 & Beca_prep_pub & $2.55 \mathrm{E}-04$ & $1.96 \mathrm{E}-04$ \\
\hline Ing2-4sm & -0.160 & $0.078^{*}$ & Beca_sup_priv & $1.71 \mathrm{E}-04$ & $8.96 \mathrm{E}-05^{*}$ \\
\hline Ing4-7sm & -0.032 & 0.087 & Beca_sup_pub & $-5.76 \mathrm{E}-05$ & $8.32 \mathrm{E}-05$ \\
\hline Ing7-10sm & 0.072 & 0.098 & Microfinanciamento & $5.32 \mathrm{E}-06$ & $6.93 \mathrm{E}-06$ \\
\hline Noahorro & -0.005 & 0.070 & Tandas & $4.56 \mathrm{E}-05$ & $2.64 \mathrm{E}-05^{*}$ \\
\hline Pareja & -0.065 & 0.091 & Fam_y_amigos & $-1.64 \mathrm{E}-05$ & $7.20 \mathrm{E}-06^{* *}$ \\
\hline Auto & -0.282 & $0.089^{* * * *}$ & Empeño & $-1.24 \mathrm{E}-05$ & $7.98 \mathrm{E}-06$ \\
\hline Teléfono & -0.158 & $0.097^{*}$ & Bancos & $1.07 \mathrm{E}-06$ & 3.79E-07*** \\
\hline Cable & -0.156 & 0.072 & Tarjetas_credito & -0.420 & $0.075^{* * *}$ \\
\hline Deuda_3001-6000 & 0.093 & $0.151^{* *}$ & Tarjetas_debito & -0.124 & $0.079^{*}$ \\
\hline Deuda_6001-15000 & 0.241 & 0.177 & Tarjetas_departamentales & 0.041 & 0.079 \\
\hline Deuda_15001-25000 & 0.420 & $0.202^{* *}$ & P_perdióempleo & 0.285 & $0.172^{*}$ \\
\hline Deuda_25001-40000 & 0.557 & $0.186^{* * *}$ & P_cambióempleo & 0.031 & 0.122 \\
\hline Deuda_40001-60000 & 0.502 & $0.197^{* *}$ & P_menorsalario & 0.493 & $0.289^{*}$ \\
\hline Deuda_mayor60000 & 0.558 & $0.192^{* *}$ & P_menoresprest & -0.007 & 0.251 \\
\hline Hmyp_priv & 0.261 & $0.097^{* * *}$ & M_perdióempleo & -0.002 & 0.121 \\
\hline Hmyp_pub & 0.106 & 0.079 & M_cambióempleo & 0.095 & 0.200 \\
\hline Hprim_priv & 0.314 & $0.062^{* * * *}$ & M_menorsalario & 0.230 & 0.269 \\
\hline Hprim_pub & 0.123 & $0.062^{* *}$ & M_menoresprest & 0.103 & 0.221 \\
\hline Hsec_priv & -0.106 & 0.082 & Int_hogar & -0.003 & 0.030 \\
\hline Hsec_pub & -0.172 & $0.078^{* *}$ & & & \\
\hline $\mathrm{n}=400$ & & & Clasificados correctament & $84.50 \%$ & \\
\hline Wald $\operatorname{chi} 2(63)=278.0$ & & & $\begin{array}{l}\text { Hosmer-Lemeshow - Goc } \\
\text { Pearson } \operatorname{chi} 2(283)=265.7\end{array}$ & ess-of-fit test - & \\
\hline Pseudo R2 = 0.5424 & & & Prob $>$ chi $2=0.7616$ & & \\
\hline
\end{tabular}

Fuente: estimación propia.

*** 0.01 .

** 0.05 .

* 0.1 .

hijos. De hecho, parece que los hogares con menores ingresos tienden a tener menos problemas para el pago de los gastos escolares, posiblemente por elegir instituciones públicas que están al alcance de su presupuesto. A pesar de ello, los aspectos relacionados con la estabilidad económica del hogar son importantes. Por cada individuo que aporta ingresos irregulares al hogar hay un $22.1 \%$ de mayor probabilidad de enfrentar problemas para pagar la educación de sus hijos, y en este mismo sentido, cuando el hogar posee activos, o usa servicios, que involucran pagos regulares para su uso (automóvil, teléfono y señal de televisión de paga), se observa una menor probabilidad de presentar este tipo de dificultades económicas (28.2, 15.8 y 15.6\%, respectivamente). 
En relación a la deuda de los hogares, respecto a los montos menores a 3,000 pesos, los rangos inmediatamente superiores (3,001-6,000 y 6,001-15,000 pesos) tampoco son estadísticamente significativos, lo cual sugiere que se trata de obligaciones financieras que los hogares logran manejar. Sin embargo, los montos mayores a 15,000 pesos se asocian fuertemente con enfrentar problemas para pagar la educación de los hijos, ya que los cambios marginales varían entre un 42 y un $55.7 \%$, dependiendo del rango de deuda.

Debido a que los costos que enfrentan los hogares están en función del número de hijos, del nivel que están cursando y del tipo de institución (privada o pública) a la cual acuden, se incluyó en el modelo el número de hijos inscritos por nivel y tipo de institución educativa.

Las estimaciones arrojaron que por cada niño inscrito en una escuela privada en el nivel de maternal o preescolar, la probabilidad de enfrentar estos problemas aumenta en un $26.1 \%$, y para aquellos que cursan la primaria en instituciones públicas o privadas la probabilidad es del $12.3 \mathrm{y}$ del $31.4 \%$, respectivamente.

De igual forma, si por un lado se considera que las instituciones privadas son más caras respecto a las públicas - lo cual se refleja en los cambios marginales-, por otro también se reconoce que la mayoría de los hogares con hijos inscritos en primaria o en los niveles inferiores pueden estar constituidos por parejas más jóvenes que podrían enfrentar mayores problemas económicos que tienden a aminorarse con el tiempo, ya que a nivel de secundaria, por cada estudiante inscrito en una institución pública la probabilidad disminuye en un 17.2\% (para los niños que cursan la secundaria en una escuela privada también se detecta una disminución en la probabilidad, pero no resulta significativa).

Aunque el estudio se enfocó en los hogares que tuvieran hijos estudiando en algún grado de educación básica, se incluyeron en el modelo variables correspondientes a niveles educativos superiores porque algunos otros integrantes estaban inscritos en estos; en general, se observa que la probabilidad de presentar problemas para cubrir los pagos relativos a la educación de los integrantes en los niveles inferiores tiende a reducirse independientemente del tipo de institución, aunque ninguna variable resultó significativa. Este resultado puede ocurrir porque solo los hogares que presentan una mejor situación económica pueden impulsar a que sus hijos sigan estudiando.

Si bien se esperaría que las becas pudieran aminorar los problemas económicos, de acuerdo a las estimaciones estas presentan una débil asociación, y únicamente para los estudiantes en los niveles de preparatoria y universidad inscritos en escuelas privadas. Estos niveles educativos ofrecidos por parte de las instituciones privadas son generalmente los más costosos, y los hogares que solicitan alguna beca lo hacen porque enfrentan dificultades económicas. En este caso, la relación positiva entre el monto de las becas y los problemas para el pago de la educación de los hijos indicaría que estos apoyos se estarían asignando a los hogares que efectivamente los necesitan.

Analizando las variables correspondientes al acceso y uso del sector financiero formal, medido a través de la posesión de alguna tarjeta de crédito y débito, la tenencia de estas permite reducir la probabilidad de tener dificultades para el pago de la educación de los hijos. En particular, el acceso a una línea de crédito se asocia con una mayor reducción $(-42 \%)$, por ser una fuente de recursos disponible en caso de necesidad. De forma similar, los ahorros, relativamente bajos de acuerdo a los resultados de la encuesta, aunque contribuyan a reducir la probabilidad, lo hacen en una menor proporción $(-12.4 \%)$.

En lo que respecta al uso, los préstamos bancarios muestran un comportamiento similar, pero reducen la probabilidad solo de forma marginal ( $0.1 \%$ por cada 1,000 pesos de financiamiento). A pesar de que la reducción sea pequeña, estos resultados sugieren que la inclusión financiera, principalmente mediante la disponibilidad de instrumentos financieros de ahorro y crédito, se 
asocia con una menor probabilidad de que el hogar enfrente problemas para pagar los gastos derivados de las actividades escolares de los hijos. Sin embargo, considerando el uso de préstamos informales, se observa que si los fondos son prestados por familiares, por cada 1,000 pesos se asocia una caída de la probabilidad del $1.64 \%$.

En relación con lo anterior, los préstamos por parte de familiares pueden ser proporcionados con mayor prontitud respecto a un banco, por lo cual pueden permitir enfrentar de manera más efectiva que los segundos las necesidades financieras del hogar. Esta interpretación también permite explicar el aumento de la probabilidad en un $4.56 \%$ si los recursos (1,000 pesos) han sido obtenidos a través de una tanda, ya que en estas el dinero se otorga según el «turno» asignado, que generalmente no coincide con el momento en el que se presenta la necesidad. Asimismo, participar en una tanda implica tener que realizar aportaciones periódicas durante toda su duración, por lo cual la obligación de un ahorro forzoso puede contribuir a los problemas económicos del hogar.

En cuanto a los cambios en el estado o las condiciones laborales, se encontró que, para el padre, el haber perdido el trabajo y seguir desempleado, o el haber encontrado otro trabajo con un menor salario durante el ciclo escolar 2010-2011, aunque sean débilmente significativos, se asocian con una elevada probabilidad de tener problemas para el pago de los gastos educativos (28.5 y $49.3 \%$, respectivamente).

Para la mayoría de los hogares, la decisión de inscribir los hijos a la escuela se toma a mediados de la primavera y se concreta algunos meses antes de que comience el año escolar (agosto-junio). Una vez inscrito(s), cambios repentinos en la estabilidad económica derivados de la pérdida del empleo — considerando los compromisos financieros adquiridos - obligan al hogar a reasignar el presupuesto familiar con el surgimiento de la problemática planteada, misma que se refleja en el modelo a través de la magnitud de los cambios marginales estimados.

Finalmente, a pesar de que la mayoría de las variables que representan los cambios en la situación laboral de la madre tengan signo positivo, ninguna de ellas resulta estadísticamente significativa, lo cual, al menos en el municipio en el cual se realizó el estudio, puede deberse a que su participación en las finanzas del hogar pudiera no ser tan relevante como la de la contraparte masculina.

\section{Conclusiones}

De acuerdo a los resultados de este trabajo, a raíz de la crisis financiera y del empleo, los hogares que tienen acceso al mercado financiero formal logran reducir de forma importante los problemas para solventar gastos en educación. Igualmente, los mecanismos de financiamiento tienden a aminorar la probabilidad de tener problemas para pagar educación, aunque se deben tomar en cuenta sus características específicas.

De esta forma, y retomando que la educación es uno de los medios mediante los cuales los individuos pueden acceder a mayores niveles de ingresos, y a sabiendas de que una mayor preparación del microempresario se asocia positivamente con las utilidades operativas de las microempresas, el acceso y el uso de los servicios financieros pueden también tener un papel importante en la acumulación de capital humano y su relación con los resultados de las unidades productivas en México.

Aunque se haya detectado la existencia de inercia en la probabilidad de tener problemas para pagar educación — lo cual deja entrever que esta dificultad no es de corto plazo—, el 
problema parece residir más en la falta de estabilidad económica que en niveles bajos de ingresos del hogar. Este resultado puede deberse a que el pago de la educación es un compromiso que la familia realiza a largo plazo, por lo que si un hogar es económicamente estable, la probabilidad de que tenga problemas para pagar los gastos educativos es menor. En este sentido, las deudas - sobre todo cuando su magnitud rebasa los 15,000 pesos- constituyen una carga financiera difícil de manejar que se asocia con mayores problemas para poder cubrir otros gastos.

Por último, se observó también que los efectos de la crisis económica se ven reflejados en una mayor probabilidad de enfrentar problemas para el pago de los gastos educativos de los hijos, en particular cuando el padre está desempleado o cuando recibe un menor salario a pesar de haberse reubicado.

\section{Una reflexión final}

Los hogares enfrentan costos de distintos tipos y magnitudes para que sus hijos/as tengan acceso a la educación y que dependen del tipo de institución (pública o privada) seleccionada. Es sabido que estudiar en una institución pública se asocia con un menor esfuerzo financiero que en una privada; sin embargo, menores costos también se asocian con menores ingresos, debido a que son los hogares con menos recursos los que suelen seleccionar la opción educativa más económica. Además de la inscripción y de la colegiatura, que probablemente constituyen la mayor diferencia entre la educación pública y la privada, hay otros los rubros comunes (útiles y uniformes). Para tales efectos, los gobiernos estatales buscan abatir estos costos proporcionando a los estudiantes de sus niveles básicos útiles y uniformes escolares gratuitos; también, otros beneficios para los estudiantes de las escuelas públicas pueden materializarse a través de tablets u otros instrumentos de aprendizaje.

Se reconocen costos que se asocian con aspectos geográficos (ruta/distancia entre la escuela y el hogar, medios de transporte y tiempos de traslado), con el modelo y la calidad (excursiones, materiales ad hoc) del servicio educativo ofertado. No menos importantes son los descuentos que ofrecen algunas escuelas privadas en la inscripción de dos o más hermanos/as, situación que además permite un ahorro de tiempo para los padres. Por último, hay costos asociados con los regalos que se realizan a los maestros en una o más ocasiones a lo largo del ciclo escolar, motivados tanto por razones sociales como por costumbre.

Debido a que la oferta educativa es particularmente dinámica, los padres están continuamente expuestos a nuevas opciones académicas diferenciadas. Considerando que la experiencia de los padres en este mercado de servicios educativos es generalmente modesta, estos se convierten en los compradores de un servicio que no consumen directamente. El escaso conocimiento en materia por parte de los progenitores puede propiciar que el estudiante se cambie de escuela o de sistema educativo, lo cual, nuevamente, repercutirá en mayores costos. Algunos de estos aspectos no han sido considerados en este trabajo, pero pueden ser la pauta para plantear nuevas hipótesis de investigación a ser analizadas en investigaciones futuras.

\section{Anexo.}

tabla A1. 
Tabla A1

Descripción de las variables independientes incluidas en el modelo

\begin{tabular}{|c|c|c|c|c|c|c|}
\hline Variable & Descripción de la variable & $\mathrm{n}$ & Media & $\mathrm{DE}$ & Min & $\operatorname{Max}$ \\
\hline $\begin{array}{l}\text { Problema2010_2011 } \\
\quad \text { (var.dep) }\end{array}$ & $\begin{array}{l}1 \text { si el hogar tuvo problemas para pagar la educación de sus hijos durante el } \\
\text { ciclo escolar 2010-2011, } 0 \text { en caso contrario }\end{array}$ & 400 & 0.363 & 0.481 & 0 & 1 \\
\hline Problema2009_2010 & $\begin{array}{l}1 \text { si el hogar tuvo problemas para pagar la educación de sus hijos durante el } \\
\text { ciclo escolar 2009-2010, } 0 \text { en caso contrario }\end{array}$ & 400 & 0.528 & 0.500 & 0 & 1 \\
\hline Problema2008_2009 & $\begin{array}{l}1 \text { si el hogar tuvo problemas para pagar la educación de sus hijos durante el } \\
\text { ciclo escolar 2008-2009, } 0 \text { en caso contrario }\end{array}$ & 400 & 0.368 & 0.483 & 0 & 1 \\
\hline Problema2007_2008 & $\begin{array}{l}1 \text { si el hogar tuvo problemas para pagar la educación de sus hijos durante el } \\
\text { ciclo escolar } 2007-2008,0 \text { en caso contrario }\end{array}$ & 400 & 0.210 & 0.408 & 0 & 1 \\
\hline P_hastaprimaria & 1 si el padre tiene escolaridad hasta el nivel de primaria, 0 en caso contrario & 400 & 0.113 & 0.316 & 0 & 1 \\
\hline Pmedia & $\begin{array}{l}1 \text { si el padre tiene escolaridad a nivel de secundaria o preparatoria, } 0 \text { en } \\
\text { caso contrario }\end{array}$ & 400 & 0.413 & 0.493 & 0 & 1 \\
\hline M_hastaprimaria & 1 si la madre tiene escolaridad hasta el nivel de primaria, 0 en caso contrario & 400 & 0.075 & 0.264 & 0 & 1 \\
\hline Mmedia & $\begin{array}{l}1 \text { si la madre tiene escolaridad a nivel de secundaria o preparatoria, } 0 \text { en } \\
\text { caso contrario }\end{array}$ & 400 & 0.568 & 0.496 & 0 & 1 \\
\hline Pes (Mes) & $\begin{array}{l}1 \text { si el padre (madre) tiene escolaridad superior, } 0 \text { en caso contrario } \\
\text { (categoría base) }\end{array}$ & & & & & \\
\hline Aport_regulares & número de personas que aportan dinero al hogar de forma regular & 400 & 1.610 & 0.748 & 0 & 6 \\
\hline Aport_irregulares & número de personas que aportan dinero al hogar de forma irregular & 400 & 0.193 & 0.448 & 0 & 2 \\
\hline Propia & 1 si la familia vive en casa/apartamento propia/o, 0 en caso contrario & 400 & 0.558 & 0.497 & 0 & 1 \\
\hline Renta & 1 si el hogar vive en casa/apartamento rentada/o, 0 en caso contrario & 400 & 0.328 & 0.470 & 0 & 1 \\
\hline Otravivienda & 1 si el hogar vive en casa de parientes, 0 en caso contrario (categoría base) & & & & & \\
\hline Ing_hasta2sm & $\begin{array}{l}1 \text { si el ingreso del hogar es menor o igual a } 2 \text { salarios mínimos }(3,158 \\
\text { pesos }), 0 \text { en caso contrario }\end{array}$ & 400 & 0.203 & 0.402 & 0 & 1 \\
\hline Ing2-4sm & $\begin{array}{l}1 \text { si el ingreso del hogar se encuentra entre } 2 \text { y } 4 \text { salarios mínimos } \\
(3,159-6,316 \text { pesos), } 0 \text { en caso contrario }\end{array}$ & 400 & 0.155 & 0.362 & 0 & 1 \\
\hline Ing4-7sm & $\begin{array}{l}1 \text { si el ingreso del hogar se encuentra entre } 4 \text { y } 7 \text { salarios mínimos } \\
(6,317-11,053 \text { pesos), } 0 \text { en caso contrario }\end{array}$ & 400 & 0.233 & 0.423 & 0 & 1 \\
\hline Ing7-10sm & $\begin{array}{l}1 \text { si el ingreso del hogar se encuentra entre } 7 \text { y } 10 \text { salarios mínimos } \\
(11,054-15,740 \text { pesos), } 0 \text { en caso contrario }\end{array}$ & 400 & 0.163 & 0.369 & 0 & 1 \\
\hline Ing_mayor10sm & $\begin{array}{l}1 \text { si el ingreso del hogar es mayor a } 10 \text { salarios mínimos (> 15,740 pesos), } \\
0 \text { en caso contrario (categoría base) }\end{array}$ & & & & & \\
\hline noahorro & 1 si el hogar no tiene ahorros, 0 si tiene ahorros & 400 & 0.513 & 0.500 & 0 & 1 \\
\hline
\end{tabular}


Tabla A1 (continuación)

\begin{tabular}{|c|c|c|c|c|c|c|}
\hline Variable & Descripción de la variable & $\mathrm{n}$ & Media & $\mathrm{DE}$ & Min & $\operatorname{Max}$ \\
\hline Pareja & $\begin{array}{l}1 \text { si en el hogar conviven ambos padres (casados o en unión libre), } 0 \text { en } \\
\text { cualquier otro caso }\end{array}$ & 400 & 0.848 & 0.360 & 0 & 1 \\
\hline Auto & 1 si el hogar cuenta con automóvil o camioneta, 0 en caso contrario & 400 & 0.635 & 0.482 & 0 & 1 \\
\hline Teléfono & 1 si el hogar cuenta con teléfono, 0 en caso contrario & 400 & 0.743 & 0.438 & 0 & 1 \\
\hline cable & 1 si el hogar cuenta con servicio de cable y 0 en caso contrario & 400 & 0.455 & 0.499 & 0 & 1 \\
\hline Deuda_menor3000 & $\begin{array}{l}1 \text { si el hogar tiene deuda menor a 3,000 pesos, } 0 \text { en cualquier otro caso } \\
\text { (categoría base) }\end{array}$ & & & & & \\
\hline Deuda_3001-6000 & 1 si el hogar tiene deuda entre 3,001 y 6,000 pesos, 0 en cualquier otro caso & 400 & 0.388 & 0.488 & 0 & 1 \\
\hline Deuda_6001-15000 & $\begin{array}{l}1 \text { si el hogar tiene deuda entre } 6,001 \text { y } 15,000 \text { pesos, } 0 \text { en cualquier otro } \\
\text { caso }\end{array}$ & 400 & 0.213 & 0.410 & 0 & 1 \\
\hline Deuda_15001-25000 & $\begin{array}{l}1 \text { si el hogar tiene deuda entre } 15,001 \text { y } 25,000 \text { pesos, } 0 \text { en cualquier otro } \\
\text { caso }\end{array}$ & 400 & 0.138 & 0.345 & 0 & 1 \\
\hline Deuda_25001-40000 & $\begin{array}{l}1 \text { si el hogar tiene deuda entre } 25,001 \text { y } 40,000 \text { pesos, } 0 \text { en cualquier otro } \\
\text { caso }\end{array}$ & 400 & 0.073 & 0.260 & 0 & 1 \\
\hline Deuda_40001-60000 & $\begin{array}{l}1 \text { si el hogar tiene deuda entre } 40,001 \text { y } 60,000 \text { pesos, } 0 \text { en cualquier otro } \\
\text { caso }\end{array}$ & 400 & 0.080 & 0.272 & 0 & 1 \\
\hline Deuda_mayor60000 & 1 si el hogar tiene deuda mayor a 60,000 pesos, 0 en cualquier otro caso & 400 & 0.068 & 0.251 & 0 & 1 \\
\hline Hmyp_priv & $\begin{array}{l}\text { número de hijos/as en institución privada a nivel maternal o preescolar } \\
\text { durante el ciclo 2010-2011 }\end{array}$ & 400 & 0.108 & 0.333 & 0 & 2 \\
\hline Hmyp_pub & $\begin{array}{l}\text { número de hijos/as en institución pública a nivel maternal o preescolar } \\
\text { durante el ciclo 2010-2011 }\end{array}$ & 400 & 0.175 & 0.424 & 0 & 2 \\
\hline Hprim_priv & $\begin{array}{l}\text { número de hijos/as en institución privada a nivel primaria durante el ciclo } \\
2010-2011\end{array}$ & 400 & 0.545 & 0.734 & 0 & 3 \\
\hline Hprim_pub & $\begin{array}{l}\text { número de hijos/as en institución pública a nivel primaria durante el ciclo } \\
\text { 2010-2011 }\end{array}$ & 400 & 0.575 & 0.810 & 0 & 4 \\
\hline Hsec_priv & $\begin{array}{l}\text { número de hijos/as en institución privada a nivel secundaria durante el } \\
\text { ciclo 2010-2011 }\end{array}$ & 400 & 0.160 & 0.458 & 0 & 2 \\
\hline Hsec_pub & $\begin{array}{l}\text { número de hijos/as en institución pública a nivel secundaria durante el } \\
\text { ciclo 2010-2011 }\end{array}$ & 400 & 0.148 & 0.414 & 0 & 2 \\
\hline Hprep_priv & $\begin{array}{l}\text { número de hijos/as en institución privada a nivel preparatoria durante el } \\
\text { ciclo 2010-2011 }\end{array}$ & 400 & 0.113 & 0.361 & 0 & 2 \\
\hline Hprep_pub & $\begin{array}{l}\text { número de hijos/as en institución pública a nivel preparatoria durante el } \\
\text { ciclo 2010-2011 }\end{array}$ & 400 & 0.108 & 0.341 & 0 & 2 \\
\hline
\end{tabular}


Tabla A1 (continuación)

\begin{tabular}{|c|c|c|c|c|c|c|}
\hline Variable & Descripción de la variable & $\mathrm{n}$ & Media & $\mathrm{DE}$ & Min & Max \\
\hline H_esc_privadas & $\begin{array}{l}\text { número de hijos/as en el hogar en instituciones de educación privad } \\
\text { durante el ciclo } 2010-2011\end{array}$ & 400 & 0.058 & 0.244 & 0 & 2 \\
\hline H_esc_públicas & $\begin{array}{l}\text { número de hijos/as en el hogar en instituciones de educación pública } \\
\text { durante el ciclo 2010-2011 }\end{array}$ & 400 & 0.050 & 0.218 & 0 & 1 \\
\hline Beca_myp_priv & $\begin{array}{l}\text { beca, en pesos, asignada a los/as hijos/as que cursan maternal o preescolar } \\
\text { en una institución privada en durante el ciclo } 2010-2011\end{array}$ & 400 & 77.38 & 637.43 & 0.00 & $7,000.00$ \\
\hline Beca_myp_pub & $\begin{array}{l}\text { beca, en pesos, asignada a los/as hijos/as que cursan maternal o preescolar } \\
\text { en una institución pública durante el ciclo 2010-2011 }\end{array}$ & 400 & 58.00 & 729.22 & 0.00 & $14,300.00$ \\
\hline Beca_prim_priv & $\begin{array}{l}\text { beca, en pesos, asignada a los/as hijos/as que cursan primaria en una } \\
\text { institución privada durante el ciclo 2010-2011 }\end{array}$ & 400 & 275.88 & 817.45 & 0.00 & $7,000.00$ \\
\hline Beca_prim_pub & $\begin{array}{l}\text { beca, en pesos, asignada a los/as hijos/as que cursan primaria en una } \\
\text { institución pública durante el ciclo 2010-2011 }\end{array}$ & 400 & 215.20 & $2,262.11$ & 0.00 & $42,900.00$ \\
\hline Beca_sec_priv & $\begin{array}{l}\text { beca, en pesos, asignada a los/as hijos/as que cursan secundaria en una } \\
\text { institución privada durante el ciclo 2010-2011 }\end{array}$ & 400 & 152.52 & $1,246.95$ & 0.00 & $14,000.00$ \\
\hline Beca_sec_pub & $\begin{array}{l}\text { beca, en pesos, asignada a los/as hijos/as que cursan secundaria en una } \\
\text { institución pública durante el ciclo 2010-2011 }\end{array}$ & 400 & 21.63 & 206.93 & 0.00 & $2,500.00$ \\
\hline Beca_prep_priv & $\begin{array}{l}\text { beca, en pesos, asignada a los/as hijos/as que cursan preparatoria en una } \\
\text { institución privada durante el ciclo } 2010-2011\end{array}$ & 400 & 100.21 & 563.03 & 0.00 & $6,000.00$ \\
\hline Beca_prep_pub & $\begin{array}{l}\text { beca, en pesos, asignada a los/as hijos/as que cursan preparatoria en una } \\
\text { institución pública durante el ciclo 2010-2011 }\end{array}$ & 400 & 20.80 & 167.02 & 0.00 & $1,850.00$ \\
\hline Beca_sup_priv & $\begin{array}{l}\text { beca, en pesos, asignada a los/as hijos/as que cursan educación superior en } \\
\text { una institución privada durante el ciclo 2010-2011 }\end{array}$ & 400 & 74.03 & 449.45 & 0.00 & $4,800.00$ \\
\hline Beca_sup_pub & $\begin{array}{l}\text { beca, en pesos, asignada a los/as hijos/as que cursan educación superior en } \\
\text { una institución pública durante el ciclo 2010-2011 }\end{array}$ & 400 & 62.90 & 595.65 & 0.00 & $8,000.00$ \\
\hline Microfinanciamento & $\begin{array}{l}\text { créditos, en pesos, otorgados al hogar por parte de instituciones } \\
\text { microfinancieras/microcrédito en los últimos } 12 \text { meses }\end{array}$ & 400 & 437.25 & $2,868.91$ & 0.00 & $48,000.00$ \\
\hline Tandas & $\begin{array}{l}\text { créditos, en pesos, otorgados al hogar por parte de una/s tanda/s en los } \\
\text { últimos } 12 \text { meses }\end{array}$ & 400 & 200.25 & 950.75 & 0.00 & $10,000.00$ \\
\hline Fam_y_amigos & $\begin{array}{l}\text { créditos, en pesos, otorgados al hogar por parte de familiares y/o amigos en } \\
\text { los últimos } 12 \text { meses }\end{array}$ & 400 & $2,134.69$ & $10,847.11$ & 0.00 & $200,000.00$ \\
\hline
\end{tabular}


Tabla A1 (continuación)

\begin{tabular}{|c|c|c|c|c|c|c|}
\hline Variable & Descripción de la variable & $\mathrm{n}$ & Media & $\mathrm{DE}$ & Min & Max \\
\hline Empeño & $\begin{array}{l}\text { créditos, en pesos, otorgados al hogar por parte casas de empeños en los } \\
\text { últimos } 12 \text { meses }\end{array}$ & 400 & 193.14 & $2,108.19$ & 0.00 & $40,000.00$ \\
\hline Bancos & $\begin{array}{l}\text { créditos, en pesos, otorgados al hogar por parte de los bancos en los } \\
\text { últimos } 12 \text { meses }\end{array}$ & 400 & $6,204.43$ & $63,073.20$ & 0.00 & $1,060,000.00$ \\
\hline Tarjetas_credito & 1 si el hogar cuenta con al menos una tarjeta de crédito, 0 en caso contrario & 400 & 0.503 & 0.501 & 0 & 1 \\
\hline Tarjetas_debito & 1 si el hogar cuenta con al menos una tarjeta de débito, 0 en caso contrario & 400 & 0.573 & 0.495 & 0 & 1 \\
\hline Tarjetas_departamentales & $\begin{array}{l}1 \text { si el hogar cuenta con al menos una tarjeta departamental, } 0 \text { en caso } \\
\text { contrario }\end{array}$ & 400 & 0.533 & 0.500 & 0 & 1 \\
\hline P_perdióempleo & $\begin{array}{l}1 \text { si el padre perdió su trabajo durante el ciclo escolar 2010-2011 y sigue } \\
\text { sin empleo, } 0 \text { en caso contrario }\end{array}$ & 400 & 0.045 & 0.208 & 0 & 1 \\
\hline P_cambióempleo & $\begin{array}{l}1 \text { si el padre perdió su trabajo y cambió de empleo durante el ciclo escolar } \\
2010-2011,0 \text { en caso contrario }\end{array}$ & 400 & 0.060 & 0.238 & 0 & 1 \\
\hline P_menorsalario & $\begin{array}{l}1 \text { si al padre no perdió su trabajo pero le redujeron el sueldo durante el } \\
\text { ciclo escolar 2010-2011, } 0 \text { en caso contrario }\end{array}$ & 400 & 0.023 & 0.148 & 0 & 1 \\
\hline P_menoresprest & $\begin{array}{l}1 \text { si al padre no perdió su trabajo pero le redujeron o quitaron prestaciones } \\
\text { durante el ciclo escolar } 2010-2011,0 \text { en caso contrario }\end{array}$ & 400 & 0.013 & 0.111 & 0 & 1 \\
\hline M_perdióempleo & $\begin{array}{l}1 \text { si la madre perdió su trabajo durante el ciclo escolar 2010-2011 y sigue } \\
\text { sin empleo, } 0 \text { en caso contrario }\end{array}$ & 400 & 0.058 & 0.233 & 0 & 1 \\
\hline M_cambióempleo & $\begin{array}{l}1 \text { si la madre perdió su trabajo y cambió de empleo durante el ciclo escolar } \\
2010-2011,0 \text { en caso contrario }\end{array}$ & 400 & 0.025 & 0.156 & 0 & 1 \\
\hline M_menorsalario & $\begin{array}{l}1 \text { si a la madre no perdió su trabajo pero le redujeron el sueldo durante el } \\
\text { ciclo escolar 2010-2011, } 0 \text { en caso contrario }\end{array}$ & 400 & 0.020 & 0.140 & 0 & 1 \\
\hline M_menoresprest & $\begin{array}{l}1 \text { si a la madre no perdió su trabajo pero le redujeron o quitaron } \\
\text { prestaciones durante el ciclo escolar } 2010-2011 \text {, y } 0 \text { en caso contrario }\end{array}$ & 400 & 0.010 & 0.100 & 0 & 1 \\
\hline Int_hogar & número de integrantes en el hogar & 400 & 4.355 & 1.266 & 1 & 11 \\
\hline
\end{tabular}

Fuente: resultados de la encuesta. 


\section{Referencias}

Bacolod, M. P. y Ranjan, P. (2008). Why children work attend school, or stay idle: The roles of ability and household wealth. Economic Development and Cultural Change, 56(4), 791-828. http://dx.doi.org/10.1086/588165

Banco de México (2011). Informe anual. México D.F. [consultado Ago 2014]. Disponible en: http://www.banxico.org.mx/ publicaciones-y-discursos/publicaciones/informes-periodicos/anual/\%7BF3075A61-2EF3-E85B-5A1CE8DE4BA3FB2C\%7D.pdf.

Baschieri, A. y Falkingham, J. (2007). Staying in School: Assessing the Role of Access, Availability and Opportunity Cost. S3RI Application and Policy Working Paper no. A07/02. University of Southampton.

Basu, K. y Van, P. H. (1998). The Economics of Child Labour. The American Economic Review, 88(3), $412-427$.

Basu, K. (1999). Child labour: Cause, consequence, and cure, with remarks on international labour standards. Journal of Economic Literature, 37(3), 1083-1119.

Becker, G. S. (1964). Human Capital: A Theoretical and Empirical Analysis with Special Reference to Education. New York, NY: Columbia University Press. http://dx.doi.org/10.7208/chicago/9780226041223.001.0001

Beegle, K., Dehejia, R. y Gatti, R. (2005). Why Should We Care about Child Labour? The Education, Labour Market, and Health Consequences of Child Labour. World Bank Policy Research Working Paper no. 3479. Washington, DC: The World Bank.

Bhalotra, S. (2007). Is child work necessary? Oxford Bulletin of Economics and Statistics, 69(1), 29-55. http://dx.doi.org/10.1111/j.1468-0084.2006.00435.x

Botello Triana, J. (2011). Algunos indicadores del mercado del trabajo. Análisis Económico, 26(63), $247-263$.

Bracho, T. (1995). Gasto privado en educación. México, 1984-1992. Revista Mexicana de Sociología, 57(2), 91-119. http://dx.doi.org/10.2307/3541071

Bray, M. (2007). The Shadow Education System: Private Tutoring and Its Implications for Planners (2nd edition). Paris: UNESCO, International Institute for Educational Planning.

Carrillo, M. (2006). La teoría y la promoción del desarrollo regional sustentable. México: Benemérita Universidad Autónoma de Puebla.

Comisión Nacional Bancaria de Valores (CNBV). (2009). Primer Reporte de Inclusión Financiera. México D.F.: CNBV.

Comisión Nacional Bancaria y de Valores (CNBV). (2010). Segundo Reporte de Inclusión Financiera. México D.F.: CNBV.

Comisión Nacional para la Protección y Defensa de los Usuarios de Servicios Financieros (CONDUSEF) (2013). El sistema financiero informal. México D.F. [consultado Ago 2013]. Disponible en: http://www.condusef.gob.mx/index.php/instituciones-financieras/otros-sectores/casas-de-empeno/519-el-sistemafinanciero-informal.

De Hoyos, R., Martínez, J. M. y Székely, M. (2010). Educación y movilidad social en México. Población, desarrollo y crecimiento. México D.F.: Centro de Estudios Espinosa Yglesias.

Edmonds, E. V. y Pavcnik, N. (2005). Child labour in the global economy. The Journal of Economic Perspective, 19(1), $199-220$.

Ersado, L. (2005). Child labour and schooling decision in urban and rural areas: Comparative evidence from Nepal, Peru, and Zimbabwe. World Development, 33(3), 455-480.

Gunnarsson, V., Orazem, P. F. y Sánchez, M. A. (2006). Child labour and school achievement in Latin America. The World Bank Economic Review, 20(1), 31-54.

Hochschild, J. L. y Scovronick, N. B. (2004). The American Dream and the Public Schools. Oxford University Press.

Instituto Nacional de Estadística y Geografía (INEGI). (2010). Encuesta Nacional de Ingresos y Gastos de los Hogares 2010. Microdatos [consultado Abril 2013]. Disponible en: http://www.inegi.org.mx/ est/contenidos/proyectos/accesomicrodatos/encuestas/hogares/regulares/enigh/2010tradicional/.

Instituto Nacional de Estadística y Geografía (INEGI), Banco de Información Económica. Encuesta mensual base 2008 de Manufactura, Comercio y Servicios, Aguascalientes, 2014.

Marcel, M. y Tokman, C. (2005). ¿Cómo se financia la educación en Chile? Santiago de Chile: Gobierno de Chile, Ministerio de Hacienda.

Mincer, J. (1974). Schooling Experience and Earnings. New York, NY: Columbia University Press.

Morrisson, C. (2002). Health, Education and Poverty Reduction. Policy Brief no. 19 OECD Development Centre. Paris: Organization for Economic Cooperation and Development.

OCDE/PISA (2000). Programa Internacional de Evaluación de los Alumnos de la OCDE [consultado Feb 2013]. Disponible en: http://www.oecd.org/pisa/keyfindings/. 
OCDE/PISA (2003). Programa Internacional de Evaluación de los Alumnos de la OCDE [consultado Feb 2013]. Disponible en: http://www.oecd.org/pisa/keyfindings/.

OCDE/PISA (2006). Programa Internacional de Evaluación de los Alumnos de la OCDE [consultado Feb 2013]. Disponible en: http://www.oecd.org/pisa/keyfindings/.

OCDE/PISA (2009). Programa Internacional de Evaluación de los Alumnos de la OCDE [consultado Feb 2013]. Disponible en: http://www.oecd.org/pisa/keyfindings/.

OECD y UNESCO (2002). (2002). Financing Education: Investments and Returns: Analysis of the World Education Indicators. Paris: OECD Publishing \& UNESCO Publishing.

Orazem, P. y Gunnarsson, V. (2003). Child Labour, School Attendance, and Performance: A Review [Working Paper]. Geneva: International Labour Office \& International. Programme on the Elimination of Child Labour.

Pavón, L. (2010). Financiamiento a las microempresas y las pymes en México (2000-2009) [Serie financiamiento del desarrollo no. 226]. Santiago de Chile: CEPAL-Naciones Unidas.

PROMÉXICO (2014). Selección de indicadores de inversión y comercio [consultado Abr 2013]. Disponible en: http://mim.promexico.gob.mx/wb/mim/seleccion_de_indicadores.

Raccanello, K. (2012). . pp. 364-390. P. Nelida Asili (Ed.), Salud, educación y nutrición: una revisión de la literatura en el marco de los Objetivos de Desarrollo del Milenio UDLAP: Vida Sustentable.

Raccanello, K. y Saucedo Carranza, C. B. (2015). Microempresas y microcréditos en la ciudad de Puebla. Perspectivas: Revista de Análisis de Economía Comercio y Negocios Internacionales, 9(1), 5-27.

Rosati, F. C. y Rossi, M. (2007). Impact of School Quality on Child Labour and School Attendance: The Case of CONAFE Compensatory Education Program in Mexico. Mexico: Understanding Children's Work (UCW) Project.

Rumberger, R. W. (1995). Dropping out of middle school: A multilevel analysis of students and schools. American Educational Research Journal, 32(3), 583-625. http://dx.doi.org/10.3102/00028312032003583

Santibáñez L., Campos M., Jarillo, B. (2011). 10 puntos para entender el gasto educativo en México: Consideraciones sobre su eficiencia. México Evalúa [consultado Mar 2013]. Disponible en: http://www.mexicoevalua.org/wpcontent/uploads/2013/02/MEX_EVA-INHOUS-GASTO_EDU-LOW.pdf.

Secretaría de Educación Pública (SEP) (2010). Sistema de Indicadores Educativos (INDISEP). México D.F.

Secretaría de Educación Pública (SEP) (2011). Sistema de Indicadores Educativos (INDISEP). México D.F.

Secretaría de Educación Pública (SEP) (2012). Principales cifras e indicadores educativos para el ciclo escolar 2011-2012. México D.F.

Secretaría de Economía (SE) (2010). Microempresas. México, D.F.: Secretaría de Economía.

Secretaría de Hacienda y Crédito Público (SHCP), Comisión Nacional Bancaria y de Valores (CNBV) e Instituto Nacional de Estadística y Geografía (INEGI) (2012). Encuesta Nacional de Inclusión Financiera 2012 (ENIF). México D.F.

Serrano, L. (1996). Indicadores de capital humano y productividad. Revista de Economía Aplicada, IV(10), $177-190$.

UNESCO (2010a). World Data on Education. VII Ed. 2010/11. UIS Statistics in Brief [consultado Feb 2013]. Disponible en: http://stats.uis.unesco.org/unesco/TableViewer/document.aspx?ReportId=121\&IF_Language=eng\&BR_Country $=4840$.

UNESCO (2010b). Informe de Seguimiento de la Educación para Todos en el Mundo [consultado Feb 2013]. Disponible en: http://www.unesco.org/new/es/education/themes/leading-the-international-agenda/efareport/

UNESCO (2011). Informe de Seguimiento de la Educación para Todos en el Mundo [consultado Feb 2013]. Disponible en: http://www.unesco.org/new/es/education/themes/leading-the-international-agenda/efareport/.

Yurén, T., de la Cruz, M., Cruz, A., Araújo-Olivera, S. S. y Estrada, M. J. (2005). Mundo de la Vida versus Habitus Escolar: El Caldo de Cultivo del Rezago Educativo en un México de Migrantes. Archivos Analíticos de Políticas Educativas, 13(13) [consultado Jun 2010]. Disponible en: http://epaa.asu.edu/epaa/v13n13/ 